\title{
(ब) $(0)$
}

(C)Auteure. Cette œuvre, disponible à

http://dx.doi.org/10.18162/fp.2020.a213, est distribuée sous licence Creative Commons Attribution 4.0 International http://creativecommons.org/licences/by/4.0/deed.fr

Marie-Pier Forest Université du Québec à Rimouski, campus de Lévis (Canada)

\section{L'effet des contextes fantaisistes sur la compréhension des élèves du premier cycle du primaire dans une approche par résolution de problèmes mathématiques}

\section{HRONIQUE • Recherche étudiante}

Cet article vise à présenter la problématique et la question de recherche d'un projet concernant l'enseignement des mathématiques à partir de contextes fantaisistes.

\section{Importance d'une approche par résolution de problèmes dans l'enseignement des mathématiques}

Selon plusieurs auteurs et chercheurs, une approche par résolution de problèmes doit être au cœur de l'enseignement des mathématiques (Kilpatrick, Swafford et Findell, 2001; National Council of Teachers of Mathematics, 2014; Pellegrino et Hilton, 2012). La résolution de problèmes serait l'une des meilleures approches pour favoriser la construction de nouvelles connaissances chez les élèves et ainsi contribuer à une véritable compréhension des mathématiques (Van de Walle et Lovin, 2007). Ainsi, les élèves résolvent des problèmes mathématiques non pas seulement pour mettre en pratique les notions qu'ils maitrisent, mais aussi, et surtout, pour en apprendre de nouvelles (DeCaro et Rittle-Johnson, 2012).

Dans les programmes scolaires actuels, au Québec et ailleurs, la résolution de problèmes présente un double rôle : elle apparait comme objet d'étude au sens où les élèves doivent apprendre à résoudre des problèmes, et elle est une approche pédagogique au sens où elle permet de construire de nouveaux concepts mathématiques (Dionne et Voyer, 2009; Fagnant et Vlassis, 2010). À travers une approche par résolution de problèmes, il est possible de mettre en place des contextes qui créent le besoin d'apprendre chez les élèves. Le contexte fait ici référence à 
la mise en situation de laquelle émerge le problème (Voyer, 2006). Il est souhaité que ces contextes suscitent l'intérêt des élèves et les incitent à se mobiliser dans la recherche de solutions au problème mathématique (Ministère de l'Éducation, du Loisir et du Sport [MELS], 2006).

\section{Évolution historique des contextes des problèmes mathématiques au Québec}

Les contextes utilisés pour présenter aux élèves des problèmes à résoudre en classe ont évolué dans le temps. Les travaux de Lajoie et Bednarz (2012) permettent de comprendre l'évolution des contextes des problèmes dans l'enseignement des mathématiques au Québec. Dans la première moitié du $20^{\mathrm{e}}$ siècle, puisque l'enseignement des mathématiques avait une visée pratique, les problèmes contenaient des données exactes à propos de la vie réelle de l'élève (Lajoie et Bednarz, 2012).

À partir des années 1980, un accent plus fort est mis sur la résolution de problèmes en classe (Dionne et Voyer, 2009). En 1988, le ministère de l'Éducation du Québec (MEQ) publie un document pédagogique, appelé Fascicule K, dans lequel la résolution de problèmes est en avant-plan. Dans celuici, il est désormais souhaité que la variété des types de contextes des problèmes mathématiques soit beaucoup plus grande. Alors que la variété reposait précédemment sur des contextes réels, on y ajoute les contextes fantaisistes (MEQ 1988). Cette nouveauté augmente la variété de problèmes pouvant être considérée dans la pratique, mais l'intention derrière celle-ci n'est pas explicite (Lajoie et Bednarz, 2012). En effet, le MEQ (1988) recommande de proposer aux élèves des problèmes avec différents types de contextes, mais sans que l'on connaisse les raisons justifiant cette recommandation.

\section{Contextes fantaisistes des problèmes mathématiques}

Par ailleurs, l'utilisation même du terme « fantaisie » porte à confusion. En effet, la nature des contextes fantaisistes est peu définie dans les programmes d'études québécois en mathématiques. La seule définition proposée est la suivante : «Un contexte est fantaisiste s'il est le fruit de l'imagination et qu'il est sans fondement dans la réalité »(MEQ 1988, p. 22). Cette définition laisse place à plusieurs interprétations, et donc à de nombreuses possibilités pour rédiger des problèmes mathématiques. $\mathrm{Si}$ l'utilisation du terme «fantaisie » est ambigüe dans le domaine des mathématiques, elle l'est également dans le domaine de la psychologie cognitive, dans lequel le terme "fantaisie » est utilisé de manière variable et est souvent vu comme un synonyme des termes "magie » et "fantastique " (Hopkins et Weisberg, 2017).

Malgré le fait que la définition de la fantaisie ne fasse pas consensus à travers les écrits scientifiques, un élément commun se retrouve dans la plupart des définitions : la fantaisie comprend des évènements qui ont peu ou pas de chances de se produire dans la réalité (Hopkins et Weisberg, 2017; Richert et Schlesinger, 2017; Weisberg et al., 2015). En mathématiques, nous remarquons que peu de recherches ont étudié les contextes fantaisistes comparativement à d'autres typologies de problèmes au regard du contexte, par exemple la dichotomie concret/abstrait et factuel/hypothétique (Caldwell et Goldin, 1979; Hembree, 1992) ou la familiarité du contexte (Palm, 2008; Vlahović-Štetić, Rovan et Mendek, 2004; Vondrová, Novotná et Havlíčková, 2019). Il s'agit d'un constat somme toute surprenant étant donné que la fantaisie est omniprésente dans la vie des enfants. Il est en effet possible de la retrouver dans les différentes sphères de leur quotidien, surtout celles associées aux divertissements (Woolley 
et Cornelius, 2013). Mais qu'en est-il de son effet sur l'apprentissage? Nous savons que les enfants apprennent des histoires dans lesquelles cohabite un mélange entre réalisme et fantaisie (Richert et Schlesinger, 2017), mais peuvent-ils réinvestir ces apprentissages dans le monde réel? Selon Hopkins et Weisberg (2017), les études en psychologie cognitive ayant traité de ce sujet obtiennent des résultats divergents qui pourraient s'expliquer par le fait que l'effet des histoires fantaisistes dépendrait de plusieurs variables, comme le type de fantaisie utilisé. Par exemple, certaines études opposent une histoire totalement réaliste à une histoire avec un degré élevé de fantaisie, cette dernière contenant plusieurs violations des lois de la nature (Walker, Gopnik et Ganea, 2015; Weisberg et al., 2015). Hopkins et Weisberg (2017) proposent donc, comme piste de recherche future, d'explorer les effets d'histoires qui distinguent plus finement le type de fantaisie.

\section{Question de recherche et méthodologie}

Cette problématique soulève une question de recherche à laquelle notre étude tentera de répondre : quel est l'effet des contextes fantaisistes sur la compréhension des élèves dans une approche par résolution de problèmes mathématiques? Afin de répondre à cette question, un devis quasi expérimental avec groupes témoin et expérimental est en développement. Ce choix nous permet d'étudier la relation entre nos deux variables ciblées, soit le recours à des contextes fantaisistes dans l'enseignement des mathématiques et la compréhension des élèves qui en émane. Ce devis aura aussi pour but de faire émerger de nouvelles questions qui pourraient être les assises d'une éventuelle suite à ce projet et qui permettraient de raffiner notre compréhension du phénomène.

\section{Conclusion}

Sur le plan scientifique, cette recherche est novatrice, car elle produira des connaissances sur une question pratiquement inexplorée en didactique des mathématiques. Les résultats contribueront à combler un manque de connaissances scientifiques au regard des contextes fantaisistes utilisés en résolution de problèmes. Sur le plan social, étant donné l'importance d'une approche par résolution de problèmes en mathématiques, il est souhaitable de connaitre de manière plus précise l'effet du degré de réalisme des contextes sur la compréhension des élèves.

\section{Références}

Caldwell, J. H. et Goldin, G. A. (1979). Variables affecting word problem difficulty in elementary school mathematics. Journal for Research in Mathematics Education, 10(5), 323-336. http://dx.doi.org/10.2307/748444

DeCaro, M. S. et Rittle-Johnson, B. (2012). Exploring mathematics problems prepares children to learn from instruction. Journal of Experimental Child Psychology, 113(4), 552-568. http://dx.doi.org/10.1016/j.jecp.2012.06.009

Dionne, J. et Voyer, D. (2009). Conférence d'ouverture : 50 ans d'enseignement des mathématiques au Québec. Bulletin $A M Q, 49(3), 6-26$. Repéré à https://archimede.mat.ulaval.ca/amq/bulletins/oct09/Conference-Dionne.pdf

Fagnant, A. et Vlassis, J. (2010). Le rôle de la résolution des problèmes dans les apprentissages mathématiques : questions et réflexions. Education Canada, 50(1), 50-52. Repéré à

https://www.edcan.ca/wp-content/uploads/EdCan-2010-v50-n1-Fagnant.pdf 
Hembree, R. (1992). Experiments and relational studies in problem solving: A meta-analysis. Journal for Research in Mathematics Education, 23(3), 242-273. http://dx.doi.org/10.5951/jresematheduc.23.3.0242

Hopkins, E. J. et Weisberg, D. S. (2017). The youngest readers' dilemma: A review of children's learning from fictional sources. Developmental Review, 43, 48-70. http://dx.doi.org/10.1016/j.dr.2016.11.001

Kilpatrick, J., Swafford, J. et Findell, B. (2001). Adding it up: Helping children learn mathematics. Washington, DC : National Academy Press. http://dx.doi.org/10.17226/9822

Lajoie, C. et Bednarz, N. (2012). Évolution de la résolution de problèmes en enseignement des mathématiques au Québec : un parcours sur cent ans des programmes et documents pédagogiques. Canadian Journal of Science, Mathematics and Technology Education, 12(2), 178-213. http://dx.doi.org/10.1080/14926156.2012.679992

Ministère de l'Éducation, du Loisir et du Sport (MELS). (2006). Programme de formation de l'école québécoise. Éducation préscolaire. Enseignement primaire. Repéré à

http://www.education.gouv.qc.ca/fileadmin/site web/documents/dpse/formation jeunes/prform2001.pdf

Ministère de l'Éducation du Québec (MEQ). (1988). Guide pédagogique. Primaire. Mathématique. Résolution de problèmes. Fascicule K. Québec, QC : Gouvernement du Québec.

National Council of Teachers of Mathematics (NCTM). (2014). Principles to actions: Ensuring mathematical success for all. Reston, VA : NCTM.

Palm, T. (2008). Impact of authenticity on sense making in word problem solving. Educational Studies in Mathematics, 67(1), 37-58. http://dx.doi.org/10.1007/s10649-007-9083-3

Pellegrino, J. W. et Hilton, M. L. (2012). Education for like and work: Developing transferable knowledge and skills in the $21^{\text {st }}$ century. Washington, DC : National Academy Press. http://dx.doi.org/10.17226/13398

Richert, R. A. et Schlesinger, M. A. (2017). The role of fantasy-reality distinctions in preschoolers'learning from educational video. Infant and Child Development, 26(4). http://dx.doi.org/10.1002/icd.2009

Van de Walle, J. A. et Lovin, L. H. (2007). L'enseignement des mathématiques : l'élève au centre de son apprentissage. Tome 1. Saint-Laurent, QC : ERPI.

Vlahović-Štetić, V., Rovan, D. et Mendek, Ž. (2004). The role of students' age, problem type and situational context in solving mathematical word problems. Review of Psychology, 11(1-2), 25-33.

Vondrová, N., Novotná, J. et Havlíčková, R. (2019). The influence of situational information on pupils' achievement in additive word problems with several states and transformations. ZDM, 51(1), 183-197.

http://dx.doi.org/10.1007/s11858-018-0991-8

Voyer, D. (2006). L'influence de facteurs liés à l'élève ou à l'énoncé sur la comprébension en résolution de problèmes écrits d'arithmétique (Thèse de doctorat, Université Laval, Québec). Repéré à https://corpus.ulaval.ca/jspui/handle/20.500.11794/18284

Walker, C. M., Gopnik, A. et Ganea, P. A. (2015). Learning to learn from stories: Children's developing sensitivity to the causal structure of fictional worlds. Child Development, 86(1), 310-318. http://dx.doi.org/10.1111/cdev.12287

Weisberg, D. S., Ilgaz, H., Hirsh-Pasek, K., Golinkoff, R., Nicolopoulou, A. et Dickinson, D. K. (2015). Shovels and swords: How realistic and fantastical themes affect children's word learning. Cognitive Development, 35, 1-14. http://dx.doi.org/10.1016/j.cogdev.2014.11.001

Woolley, J. D. et Cornelius, C. A. (2013). Beliefs in magical beings and cultural myths. Dans M. Taylor (dir.), The Oxford bandbook of the development of imagination (p. 61-74). New York, NY : Oxford University Press.

http://dx.doi.org/10.1093/oxfordhb/9780195395761.013.0005

\section{Pour citer cet article}

Forest, M.-P. (2020). L'effet des contextes fantaisistes sur la compréhension des élèves du premier cycle du primaire dans une approche par résolution de problèmes mathématiques. Formation et profession, 28(3), 115-118.

http://dx.doi.org/10.18162/fp.2020.a213 